\title{
Rituximab promising for idiopathic membranous nephropathy
}

Rituximab seems to be a safe and effective treatment for high-risk patients with idiopathic membranous nephropathy, show researchers in Italy. "We initially tested the safety of rituximab in eight patients with membranous nephropathy and severe nephrotic syndrome unresponsive to angiotensin-convertingenzyme inhibition", say investigators Piero Ruggenenti and Giuseppe Remuzzi. "Treatment was well tolerated and led to a significant reduction in proteinuria so we decided to consider rituximab as first-line therapy for those patients with persistent nephrotic syndrome despite optimized conservative therapy."

\section{Rituximab was effective even when previous treatment with other immunosuppressive drugs had failed... 77}

Patients with idiopathic membranous nephropathy and persistent proteinuria who were predicted to progress to end-stage renal disease or die prematurely from disease-related complications $(n=100)$ received rituximab and were followed up for at least 6 months (median follow-up 29 months). Overall, 65 patients achieved the primary end point of complete remission (24h proteinuria $<0.3 \mathrm{~g})$ or partial remission $(24 \mathrm{~h}$ proteinuria $<3 \mathrm{~g}$ with a $>50 \%$ reduction in proteinuria from baseline). Treatment effect was time dependent and all patients with at least 4 years of follow-up achieved complete or partial remission. Of the 35 patients who did not reach the end point, 20 had decreased proteinuria to $<50 \%$ of baseline values. "Rituximab was effective even when previous treatment with other immunosuppressive drugs had failed", highlight the investigators.

In patients with complete or partial remission, rituximab resulted in an increase in glomerular filtration rate (GFR) and serum albumin levels, decrease in albumin fractional clearance, amelioration of dyslipidaemia, decrease in circulating $\mathrm{B}$ lymphocytes and a reduced risk of cardiovascular complications. Female sex, lower $24 \mathrm{~h}$ urinary protein excretion and lower serum creatinine levels at inclusion predicted a higher rate of disease remission and more proteinuria reduction on follow-up. Larger proteinuria reduction predicted a slower decline in GFR. Only nonserious, transient treatment-related adverse events were reported.

"We will continue to follow all our patients to assess whether complete or partial remission achieved by rituximab translates into long-term protection from progression to kidney failure and need of dialysis and transplantation and, even more importantly, protection from myocardial infarction, stroke and other potentially fatal complications of the nephrotic syndrome", conclude Ruggenenti and Remuzzi.

Helene Myrvang

Original article Ruggenenti, P. et al. Rituximab in idiopathic membranous nephropathy. J. Am. Soc. Nephrol. doi:10.1681/ASN.2012020181 